\title{
MedienPädagogik
}

www.medienpaed.com

Zeitschrift für

Theorie und Praxis

der Medienbildung

ISSN 1424-3636

Themenheft Nr. 22: Frühe Medienbildung

\section{Medienerziehung in Kindertagesstätten Nachhaltigkeitsüberlegungen im Anschluss an eine Fortbildungsinitiative}

Henrike Friedrichs und Dorothee M. Meister

\begin{abstract}
Durch die Einführung und Aktualisierung der Bildungspläne sind Kindertagesstätten seit einigen Jahren vermehrt aufgefordert, medienerzieherisch aktiv zu werden. Auch wenn es inzwischen verschiedene Fortbildungsinitiativen gibt, können aktuelle Studien keine grundlegenden Fortschritte in einer flächendeckenden praktizierten Medienerziehung nachweisen. Im vorliegenden Beitrag wird mit Bezug auf empirische Studien und strukturelle Rahmenbedingungen die aktuelle Situation der Medienerziehung in Deutschland und spezifisch in Nordrhein-Westfalen aufgezeigt. Daran anknüpfend stellen die Autorinnen ein Fortbildungskonzept der medienpädagogischen Initiative Medienkompetenz-Kitas NRW vor, das mittels eines Inhouse-Schulungsansatzes in zwölf Kindertagesstätten in NRW durchgeführt wurde. Im Fokus der Fortbildung standen die Vermittlung technischen Grundlagenwissens und handlungsorientierter Methoden für eine aktive medienpraktische Arbeit sowie die Förderung medienpädagogischen Reflexionswissens. Auf der Grundlage der Evaluation des Projekts und der aktuellen Situation der Medienerziehung werden im Zuge einer angestrebten nachhaltigen Verankerung von Medienerziehung in Kindertagesstätten Implikationen zur Fort- und Ausbildung von Erzieherinnen und Erziehern benannt.
\end{abstract}

\section{Mediatisierte Kindheit}

Die Mediatisierung der Lebenswelt erreicht Kinder in einem immer früheren Lebensalter. In Familien mit Kindern im Vorschulalter ist die Medienausstattung bereits breit gefächert (mpfs 2012; mpfs 2015; Aufenanger 2013; Egmont Ehapa 2013; Feierabend und Klingler 2014; Institut für Demoskopie Allensbach 2015). Laut der quantitativen Studie miniKIM 2014 wachsen fast alle Kinder im Alter von zwei bis fünf Jahren in Deutschland in einem Haushalt mit einem Fernsehgerät, einem Computer bzw. Laptop, einem Handy bzw. Smartphone und einem Radio auf. Nur 5 Prozent der Haushalte verfügen nicht über einen Internetzugang. In 40 Prozent der Familien sind zudem eine Spielekonsole und in 23 Prozent ein Tablet und ein Kindercomputer vorhanden (mpfs 2015, 5). Insofern nutzen schon Vorschulkinder jenseits der klassischen Bilderbücher ein breites Spektrum an Medien. So sind (Bilder-) Bücher für Kinder im Alter von zwei bis drei Jahren zwar noch das wichtigs-

Friedrichs, Henrike und Dorothee M. Meister. 2015. «Medienerziehung in Kindertagesstätten - Nachhaltigkeitsüberlegungen im Anschluss an eine Fortbildungsinitiative.» MedienPädagogik 22 (28. Mai): 1-23. https://doi.org/10.21240/ mpaed/22/2015.05.28.X. 
te Medium, bei den Vier- bis Fünfjährigen hingegen stellt der Fernseher bereits das Lieblingsmedium dar (mpfs 2015, 9, 12; Aufenanger 2013, 13; Institut für Demoskopie Allensbach 2015, 5). Durch die alltägliche Konfrontation mit Mediengeräten entstehen auch kindliche Besitzwünsche. Etwa ein Drittel der Vier- bis Fünfjährigen wünscht sich laut KidsVerbraucherAnalyse 2013 einen eigenen Fernseher, ca. 30 Prozent ein Smartphone und etwa 22 Prozent einen Computer bzw. Laptop (Egmont Ehapa 2013, 72).

Angesichts der alltäglichen Mediennutzung von Kindern sind kontroverse (pädagogische) Diskussionen darüber entbrannt, welche Konsequenzen sich durch die Vielzahl an medial vermittelten Angeboten für Kinder ergeben. Auf der einen Seite werden etwa die Lernpotenziale positiv hervorgehoben, da Kinder über Medien ihre Lebenswelt erweitern, Interessen vertiefen und sich Wissen (spielerisch) einprägen könnten (Aufenanger und Gerlach 2005; Götz 2010). Auch für die Identitätsentwicklung können Medien eine wichtige Ressource bieten, etwa indem Kinder mittels Mediennutzung Entwicklungsthemen bearbeiten und sich in einer identifikatorischen Weise Medienfiguren zum Vorbild nehmen (z.B. indem sich ein schüchternes, zurückhaltendes Kind an einer mutigen Medienfigur orientiert) (Hoffmann 2004; Krotz 2003; Rogge 2007). Auf der anderen Seite werden mit der Rezeption von Medieninhalten mögliche Gefährdungspotenziale für die kindliche Sozialisation verknüpft: Medienkritiker/innen befürchten, dass der kindliche Medienkonsum mit Bewegungsmangel einhergehe (Pfeiffer 2011), das handelnde, kreative Erleben (Hentig 1987) und das Unterscheidungsvermögen von 〈realer〉 und 〈virtueller〉 Welt reduziere oder gar zur Mediensucht führe (Spitzer 2012). Risiken bestehen zudem darin, dass Medieninhalte Ängste bei Kindern entstehen lassen können (Rogge 2003) oder Aggression und gewalthaltiges Verhalten durch gewalthaltige Medieninhalte unter spezifischen negativen Sozialisationsbedingungen gefördert werden kann (Kunczik und Zipfel 2013; Möller und Krahé 2013). Gerade die vielfältigen Inhalte und Leitbilder der kommerziellen Angebote (vgl. Livingstone und Helsper 2006; Meister et al. 2011; Dreyer et al. 2014; Vollbrecht 2010; Woldemichael 2010; Bachmair 2002, 13) sowie geschlechterstereotype oder sonstige stereotype Inhalte (Becker 2015; Luca 2010; Götz und Lemish 2012; Götz 2014) können, so eine weitere Befürchtung, möglicherweise unhinterfragt übernommen werden.

\section{Medienerziehung in Kindertagesstätten}

Aufgrund der medialen gesellschaftlichen Durchdringung und fortwährender Medienentwicklungen sind Kindertagesstätten neben dem Elternhaus in der Verantwortung, das Thema Medien in ihrer pädagogischen Arbeit aufzugreifen (Eder und Roboom 2014). Medienerziehung, verstanden als «Erziehung zur reflektierten Mediennutzung» (Schorb 2005, 241), ist bereits bei Kindern im Vorschulalter uner- 
lässlich, um negative Einflüsse von Medien zu kanalisieren, ihre positiven Wirkpotenziale zu stärken und kindliche Medienkompetenzen umfassend zu fördern (Baacke 1997, 98f.). Eine solche Medienerziehung darf nicht bei einem unreflektierten euphorischen Einsatz von Medien oder blossen Verboten stehenbleiben. Stattdessen sollten Erzieher/innen sowohl Argumentationen für und gegen den Gebrauch elektronischer Medien in der Kita berücksichtigen - hierzu benötigen sie fundierte Kenntnisse sowohl zur kindlichen Mediennutzung und damit verbundenen Chancen und Risiken als auch über medienerzieherische Handlungsmöglichkeiten.

Medienpädagogen/innen aus Wissenschaft und Praxis haben verschiedene Konzepte zur Medienerziehung in der Kindertagesstätte entwickelt und auch politische Forderungen formuliert. Zu benennen ist hierbei u.a. die Initiative Keine Bildung ohne Medien! (KBoM!), deren zentrales Anliegen eine mediale Grundbildung aller pädagogischen Fachkräfte ist (KBoM! 2014).

Fragt man sich, wie Medienerziehung in institutionellen Bildungskontexten verankert werden kann, so deutet viel darauf hin, dass die vorschulische Bildung ähnlich wie die schulische Medienerziehung zu behandeln ist (Meister 2013). So bedarf es nicht nur engagierten und qualifizierten Personals, sondern Grundvoraussetzung sind entsprechende Rahmenbedingungen. Hierzu zählen einrichtungsinterne Bedingungen wie vorhandene medienpädagogische Konzepte in den Einrichtungen und eine adäquate Medienausstattung sowie ausreichende zeitliche und personelle Ressourcen aufseiten der Erzieher/innen. Zum anderen müssen stimmige externe strukturelle Bedingungen vorliegen: Die (gesetzlichen) Rahmenbedingungen der Arbeit in der Kita sollten eine Umsetzung ermöglichen und der Bereich der Medienerziehung gehört als fester Bestandteil in die Aus- und Fortbildung von Erzieher/innen verankert (Friedrichs 2013, 10ff.).

\section{Die Bildungspläne}

In den Bildungsplänen der Bundesländer, welche die Grundlage für die pädagogische Arbeit der Kindertagesstätten und teilweise auch Grundschulen darstellen, wird explizit die Erwartung an Erzieher/innen formuliert, den Medienbereich in ihrer pädagogischen Arbeit zu berücksichtigen. Die Art und Verankerung der medienpädagogischen Leitvorstellungen variieren in den verschiedenen Bildungsplänen. In den meisten Bildungsplänen stehen das Erlernen eines sachgerechten Umgangs mit Medien, eine technische Nutzung sowie Möglichkeiten der Mediendidaktik im Fokus, nur in wenigen Plänen werden die gestalterischen und kreativen Möglichkeiten der Medienarbeit als Aufgaben der frühkindlichen Bildung benannt. Zwar wird der Bereich Medien mittlerweile in allen Bildungsplänen aufgegriffen, jedoch selten als separater Bildungsbereich (Friedrichs und Meister 2015, 290f.; Neuß 2013, 36f.). Da die Fortbildungsinitiative Medienkompetenz-Kitas NRW, die in diesem Beitrag thematisiert wird, in Nordrhein-Westfalen stattgefunden hat, 
folgt ein kurzer Exkurs zu dem dort geltenden Bildungsplan: In den Grundsätzen zur Bildungsförderung für Kinder von 0 bis 10 Jahren in Kindertageseinrichtungen und Schulen im Primarbereich in Nordrhein-Westfalen, die im Mai 2010 durch das Ministerium für Schule und Weiterbildung des Landes Nordrhein-Westfalen und das Ministerium für Familie, Kinder, Jugend, Kultur und Sport des Landes Nordrhein-Westfalen herausgegeben wurden, werden zehn Bildungsbereiche benannt: Bewegung; Körper, Gesundheit und Ernährung; Sprache und Kommunikation; soziale, kulturelle und interkulturelle Bildung; musisch-ästhetische Bildung; Religion und Ethik; mathematische Bildung; naturwissenschaftlich-technische Bildung; ökologische Bildung und Medien. Ein besonderes Merkmal des Bildungsplans von NRW ist somit die Nennung von Medien als expliziter Bildungsbereich. Das Ziel einer frühen Medienbildung sei es, die "Mensch-Medien-Interaktion verantwortungsvoll einzuschätzen und entwicklungsfördernd einzusetzen» (MSW NRW und MGFFI NRW 2010, 67), sodass Kinder sich zu medienkompetenten Persönlichkeiten entwickeln. Zwar liefert der Bildungsplan Anregungen und Beispiele produktionsorientierter, reproduktionsorientierter und rezeptiver Medienarbeit (z.B. eine Auseinandersetzung mit den medialen Lieblingsfiguren der Kinder, PC-Kurse etc.), Informationen zur detaillierteren Projektkonzeption sind jedoch nicht gegeben. Dementsprechend bedarf es aufseiten der Erzieher/innen weiterer Vorbereitung und Informationsbeschaffung, um ein medienpädagogisches Projekt umzusetzen. Informationen hinsichtlich einer altersentsprechenden Mediennutzung und Vermittlung von Medienkompetenz liegen ebenfalls nicht vor (ebd., 66ff.; Friedrichs und Meister 2015, 292f.). Dies wäre jedoch aus zweierlei Gründen wünschenswert: Zum einen umfasst die pädagogische Arbeit in der Kindertagesstätte eine breite Altersspanne von Kindern und zum anderen richtet sich der Bildungsplan sowohl an die Kindertagesstätte als auch an die Grundschule, sodass die Verantwortung nicht klar definiert wird und es somit zu einer Verantwortungszuweisung der Kita an die Grundschule bzw. die Offene Ganztagsschule kommen kann.

Verankerung medienpädagogischer Inhalte in der Erzieher/innen-Ausbildung Friedrichs (2013) analysierte im Rahmen ihrer Dissertation u.a. die Struktur und Verankerung medienpädagogischer Inhalte in der Erzieher/innen-Ausbildung in Nordrhein-Westfalen. Hierzu wertete sie die Richtlinien für die Fachschulen des Sozialwesens mit der Fachrichtung Sozialpädagogik in Nordrhein-Westfalen aus und führte sowohl sechs leitfadengestützte Interviews mit Lehrkräften des Faches Sprache(n)/Medien als auch acht Gruppendiskussionen mit angehenden Erzieher/ innen, die sich im zweiten Ausbildungsjahr bzw. im Berufspraktikum (drittes Ausbildungsjahr) in den Einrichtungen befinden.

Zum Zeitpunkt der Erhebung (09/2011-06/2012) erfolgte der medienpädagogische Unterricht an den Fachschulen im Fach Sprache(n)/Medien, welches an den Fach- 
schulen mit zwei Unterrichtsstunden pro Woche unterrichtet wird (siehe auch Six und Gimmler 2007a, 68ff.). Die zeitliche und inhaltliche Ausgestaltung des Faches Sprache(n)/Medien variiert insofern an den Fachschulen, als an manchen Schulen im ersten Ausbildungsjahr nur der Bereich Kinderliteratur (z.B. Bilderbuchbetrachtung) betrachtet wird und erst im zweiten Ausbildungsjahr der Blick auf elektronische Medien gelegt wird, während an anderen Schulen in beiden Ausbildungsjahren die Bereiche inhaltlich verknüpft werden. Im Hinblick auf die Ausbildungsinhalte geben alle Lehrkräfte des Samples an, in ihrem Sprache(n)/Medien-Unterricht theoretisch-reflexive Inhalte (z.B. Themen wie Kommerzialisierung, pädagogische Einschätzung von Kindersendungen etc.) zu behandeln sowie produktionsorientierte Medienarbeit (z.B. Erstellung von Videos) umzusetzen. Die medienpädagogische Umsetzung bleibt jeweils auf den schulischen Kontext beschränkt, sodass nur diejenigen Auszubildenden, die sich explizit im Rahmen einer Projektarbeit für den Medienbereich entscheiden, vertiefte medienpädagogische Praxiserfahrungen mit Kindern sammeln (Friedrichs 2013, 10).

Während im Lehrplan aus dem Jahr 2010 Sprache(n)/Medien einen von vier Bildungsbereichen der Kinder- und Jugendarbeit darstellt, wird Sprache(n)/Medien in den aktualisierten Richtlinien der Erzieher/innen-Ausbildung, die zum Schuljahr 2014/15 in Kraft getreten sind, nicht mehr als separater Bildungsbereich genannt, sondern ist im Lernbereich Sozialpädagogische Bildungsarbeit in den Bildungsbereichen professionell gestalten als einer der zehn Bildungsbereiche, die im Entwurf zu den Grundsätzen zur Bildungsförderung für Kinder von 0 bis 10 Jahren in Kindertageseinrichtungen und Schulen im Primarbereich in NordrheinWestfalen festgelegt sind, verankert. Das zur Verfügung stehende Stundenkontingent für Sprache(n)/Medien ist nicht explizit festgeschrieben, da nur Angaben für den gesamten Lernbereich gemacht werden (MSW NRW 2014, 50ff.). Weiter wird die «Vermittlung von Medienkompetenz» (ebd.,18) als Querschnittaufgabe innerhalb der Ausbildung verstanden. Im Kontext des neuen Lehrplans modifizieren die Fachschulen ihren Unterricht und somit auch die medienpädagogischen Ausbildungsinhalte. Hiermit ist ein deutliches Risiko verbunden, dass der Bereich Medienerziehung zukünftig weniger Raum einnimmt. So berichtete ein Lehrer des Samples in der Studie von Friedrichs $(2013,13)$, dass Sprache(n)/Medien an der Fachschule zukünftig nicht mehr als alleiniges Fach unterrichtet werde, sodass das Zeitpensum, dass für den Bereich verwendet wird, bei der Berücksichtigung der insgesamt zehn Bildungsbereiche geringer ausfallen dürfte.

\section{Ist-Stand der Medienerziehung in der Kindertagesstätte}

Auch wenn Medienerziehung in Kindertagesstätten mittlerweile von der Politik als Bildungsbereich aufgegriffen wird, bedeutet dies noch nicht, dass Medienerziehung einen hohe subjektive Wichtigkeit für Erzieher/innen hat und dass regelmäs- 
sig medienpädagogisch mit den Kindern in den Einrichtungen gearbeitet wird: Eine aktuelle repräsentative Studie des Instituts für Demoskopie Allensbach (2015, 30) verdeutlicht, dass digitale Medien selten in der Kita eingesetzt werden: Nur 14 Prozent der Erzieher/innen in Deutschland integrieren mindestens ab und zu den Computer und andere digitale Medien in die pädagogische Arbeit, knapp die Hälfte der Erzieher/innen nie (Institut für Demoskopie Allensbach 2015, 30). Eine Studie von Meister et al. (2012, 23f.), welche eine Online-Repräsentativbefragung in den Kindertagesstätten in NRW durchgeführt haben, zeigt auf, dass auch in Nordrhein-Westfalen Computer und Internet in den meisten Einrichtungen nur selten mit den Kindern genutzt werden. Stattdessen werden neben dem digitalen Fotoapparat vor allem auditive Medien wie Kassettenrekorder und CD-Player verwendet, um Musik oder Hörspiele anzuhören. Demzufolge ist der Medieneinsatz in der Kita nach wie vor "grösstenteils an den für Kindertagesstätten klassischen Medien» (Brüggemann et al. 2013, 24) wie Bilderbüchern (ebd., 23) oder den eben genannten elektronischen Medien ausgerichtet.

Zurückzuführen ist die geringe Nutzung neuer Medien in den Einrichtungen auf Ansichten der Erzieher/innen, die einer Auseinandersetzung mit (digitalen) Medien wie dem Computer entgegenstehen. Hierzu zählen negative Wirkannahmen im Hinblick auf die Nutzung digitaler Medien (PC, Tablet, Smartphone) und des Fernsehens durch Kinder wie eine Überforderung durch die Informationsflut, eine Verkümmerung der Talente (Institut für Demoskopie Allensbach 2015, 62) und eine Aggressionssteigerung (Six und Gimmler 2007a, 173ff.). Zudem lehnen Erzieher/innen die Nutzung digitaler bzw. elektronischer Medien für die Kita ab, da sie ohnehin einen hohen Medienkonsum auf Seiten der Kita-Kinder wahrnehmen wird (Institut für Demoskopie Allensbach 2015, 62; Meister et al. 2012, 21), dies gilt vor allem für Kinder aus problembelasteten Familien (Six und Gimmler 2007a, 173ff.). Weiter sehen sich die Erzieher/innen für andere Bildungsbereiche und -erfahrungen (z.B. Naturkenntnisse, Sprachförderung) stärker verantwortlich als für Medienerziehung und eine Heranführung an Medien (Institut für Demoskopie Allensbach 2015, 58; Six und Gimmler 2007a, 203ff.). Die Sorge, dass durch den Einsatz digitaler Medien in der Kita die Kinder von den Inhalten, welche die Erzieher/innen vermitteln möchten, abgelenkt werden (Institut für Demoskopie Allensbach 2015, 58), dürfte ebenfalls eine Ablehnung der Integration dieser Medien in die Kita-Arbeit begünstigen. Diese eher bewahrpädagogischen Haltungen sind nicht generationsspezifisch: Die Analyseergebnisse der Dissertation von Friedrichs (2013) zum medienerzieherischen Habitus angehender Erzieher/innen, die sich noch in der Ausbildung befinden, zeigen auf, dass solche eher Haltungen auch bei jungen Pädagogen/innen, die privat sehr medienaffin sind, vorhanden sind und nicht nur bei älteren und eventuell technisch weniger versierteren Erzieher/innen. 
Obwohl die Erzieher/innen dem Einsatz digitaler Medien in der Kita überwiegend kritisch gegenüberstehen (Institut für Demoskopie Allensbach 2015, 53ff.), befürworten die meisten Erzieher/innen in NRW Medienerziehung im Sinne der Förderung eines verantwortungsvollen kindlichen Medienumgangs (Six und Gimmler 2007a, 202). Dies ist jedoch nicht per se an ein Interesse der Erzieher/innen für die Thematik gekoppelt: Während 62 Prozent der Befragten (grösstenteils KitaLeitungen) in der Studie von Meister et al. (2012) das Interesse der Kita-Leitung der Einrichtung für Medienerziehung als sehr hoch bzw. hoch einschätzen, nehmen dies nur 29 Prozent der Befragten für die Erzieher/innen wahr (ebd., 21f.). Etwa ein Drittel schätzt das Interesse der Erzieher/innen als (eher) niedrig ein (ebd., 19). Dennoch ist, wie aufgezeigt, eine Aufgeschlossenheit für die Förderung kindlicher Medienkompetenzen vorhanden, wenngleich diese kaum Auswirkungen auf die erzieherische Praxis hat: Produktionsorientierte Medienarbeit wie bspw. die Erstellung von digitalen Bilderbüchern in den Einrichtungen findet in den Einrichtungen in Nordrhein-Westfalen kaum statt. Eine Ausnahme stellen Medienprodukte im auditiven Bereich dar, die etwas häufiger Verwendung finden, was mit der im Kinderbildungsgesetz verankerten Sprachförderung der Kinder verknüpft sein dürfte (KiBiz §13). Zudem haben die digitale Fotografie und auch das Erstellen von Videos zwar Einzug in die Kitas gefunden, der Medieneinsatz scheint jedoch oftmals auf die Dokumentation der kindlichen Entwicklung beschränkt zu sein (welche ebenfalls durch das KiBiz gesetzlich gefordert wird). Weiter finden zwar medienbezogene Gespräche mit Kindern statt (Meister et al. 2012, 23f.), dies geschieht jedoch vor allem als Reaktion auf kindliche Fragen und Verhaltensweisen der Kinder und wird nicht aktiv durch die Erzieher/innen angeregt (Six und Gimmler 2007a, 230f.). Obwohl der Bereich der medienpädagogischen Elternarbeit von den Erzieher/innen als wichtig betrachtet wird, findet kaum ein diesbezüglicher Austausch mit den Eltern statt. Dies dürfte u.a. damit zusammenhängen, dass Gespräche mit den Eltern zur kindlichen Mediennutzung teilweise als schwierig und heikel wahrgenommen werden (Meister et al. 2012, 32; Six und Gimmler 2007, 259ff.).

Neben den genannten Kognitionen der Erzieher/innen nehmen die Erzieher/innen eine mangelnde Geräteausstattung (Meister et al. 2012, 19), knappe zeitliche Ressourcen (Meister et al. 2012, 19), ein begrenztes Wissen der Erzieher/innen zum Bereich der Medienerziehung (Six und Gimmler 2007a, 192ff.; Institut für Demoskopie Allensbach 2015, 36ff.) als hinderlich für eine Umsetzung von Medienerziehung in den Einrichtungen wahr. Hinzu kommen ein geringes Vertrauen der Erzieher/innen in ihre eigene Medien- und medienpädagogische Kompetenz (Meister et al. 2012, 19f., 22; Six und Gimmler 2007a, 132ff., 148ff.). So schätzen etwa 34 Prozent der Befragten der Studie von Meister et al. (2012) die technische Medienkompetenz der Erzieher/innen als (eher) gering ein (ebd., 19f.). Daraus resultierend bestehen Unsicherheiten im Hinblick auf das Selbstvertrauen der Erzieher/innen, selbstständig Medienprojekte durchzuführen (ebd., 22). 


\section{Projektkonzeption und Evaluationskonzept von Medienkompetenz-Kitas NRW}

Angesichts der nach wie vor wenig etablierten Medienerziehung in den Kitas besteht noch immer ein hoher Bedarf nach Unterstützung der medienerzieherischen Praxis. Bisherige Ansätze setzten zumeist auf zentrale Fortbildungen oder auf die Unterstützung durch Handreichungen, die in der Fläche ganz offenbar nicht den Erfolg zeigten, den man sich erhofft hat. So untersuchte das Forschungsteam um Ulrike Six bereits zweimal die Situation der Medienerziehung in Kitas in NordrheinWestfalen (Six et al. 1998; Six und Gimmler 2007a). Laut den Ergebnissen der ersten der beiden Repräsentativstudien fanden Mitte der 1990er Jahre kaum medienpädagogische Aktivitäten in den Einrichtungen statt und die Kenntnisse der Erzieherinnen im Bereich der Medienerziehung wurden als defizitär eingestuft. Infolgedessen entstanden vielfältige medienpädagogische Fortbildungsangebote und Informationsmaterialien, die jedoch, wie die zweite Repräsentativstudie von Six und Gimmler (2007a) zeigt, auch beinahe 10 Jahre später nicht ausreichend genutzt wurden und vielfach noch immer wenig bekannt waren.

Mit Medienkompetenz-Kitas NRW. Initiative zur Medienkompetenzförderung in nordrhein-westfälischen Kindertagesstätten sollten nun neue Wege bei einer medienpädagogischen Fortbildung beschritten werden ${ }^{1}$. Aufgegriffen wurden in der Konzeption Anregungen von Six und Gimmler (2007a, 290ff.), welche aufzeigen, dass es für eine Umsetzung von Medienerziehung in Kindertagesstätten zentral ist, dass die Erzieher/innen ein adäquates Verständnis von Medienerziehung haben, Medienerziehung als wichtig einschätzen und sich zu einer Umsetzung befähigt sehen (Six und Gimmler 2007a, 266).

Die Fortbildung sollte auf die Teams in den Einrichtungen ausgerichtet sein und einen «niederschwelligen` Ansatz der Medienkompetenzvermittlung verfolgen. Dies wurde derart gestaltet, dass Inhouse-Schulungen mit dem gesamten Kollegium einer Kita durch eine/n erfahrene/n Medienpädagogen/in durchgeführt wurden und, basierend auf einem systemischen Ansatz, kooperative und selbstorganisierte Lernformen aufgebaut werden sollten. Mit dem Ansatz sollte zudem sichergestellt werden, dass die Technik vor Ort in die Schulung einbezogen wurde, um Praxisnähe und Transfersicherung besser zu gewährleisten. Schliesslich ging es auch darum, das Team über einen längeren Zeitraum zu begleiten, um so die Bandbreite an Praxismöglichkeiten aufzuzeigen und eine nachhaltige Verankerung von Medienerziehung in den Einrichtungen zu erzielen (Eder et al. 2014).

An dem Fortbildungskonzept nahmen insgesamt zwölf Kindertagesstätten in NRW teil. Die Inhouse-Schulungen fanden durch Medienpädagogen/innen statt, die be-

1 Das Projekt wurde im Auftrag der Landesanstalt für Medien (LfM) von Mai 2010 bis Juni 2011 von den Projektpartnern Schulen ans Netz e.V. (Projektkoordination) und Blickwechsel e.V. (Qualifizierung und Begleitung der zuständigen Medienpädagogen/innen) durchgeführt und durch die Gesellschaft für Medienpädagogik und Kommunikationskultur (GMK) extern evaluiert. Verantwortlich für die Evaluation waren Prof. Dr. Dorothee Meister (Universität Paderborn), Henrike Friedrichs (Universität Bielefeld) und Jürgen Lauffer (Geschäftsführer der GMK) 
reits medienpädagogisch erfahren waren und im Vorfeld durch die medienpädagogische Institution Blickwechsel e.V. speziell für das Projektvorhaben in einer zweitägigen Schulungsmassnahme fortgebildet wurden. Insbesondere wurden verschiedene Möglichkeiten der Vermittlung von medienpädagogischen Ideen an die Erzieher/innen und zur Stärkung der technischen Bedienkompetenz auf Erzieher/innen-Seite erarbeitet.

Die Medienpädagogen/innen begleiteten die Kitas jeweils für ein halbes Jahr in ihrer medienpädagogischen Arbeit. Je nach Bedarf konnten die Einrichtungen ihre Fortbildungstage flexibel wählen, diese war jedoch auf maximal acht halbe Tage zu verteilen. Es fanden Schnuppertage statt, an denen die Erzieher/innen ohne das Beisein von Kindern geschult wurden, sowie medienpädagogische Thementage, an denen die Erzieher/innen, begleitet durch die Medienpädagogen/innen, die erlernten medienpädagogischen Inhalte mit den Kindern umsetzen konnten (Eder et al. 2014, 18). In Absprache mit den Kitas wurden die Fortbildungsinhalte an die Bedürfnisse der jeweiligen Einrichtung angepasst und ein jeweils individuelles Fortbildungskonzept erstellt. Trotz des zielgruppenspezifischen Zuschnitts der Fortbildung in den einzelnen Kitas sind Schwerpunkte erkennbar, die in fast allen Einrichtungen umgesetzt wurden. Hierzu zählen Themen wie: Sensibilisierung für Medienerziehung, Thematisierung von Medienhelden/innen², medienpraktische Arbeit in den Bereichen Audio, digitale Fotografie, Video und Computer. Als praktische Methode im Bereich Audio wurde zum Beispiel eine GeräuscheRallye durchgeführt, bei der Kinder Geräusche in der Kita aufnahmen, welche die anderen Kinder erraten sollten. Im Bereich Video wurde unter anderem mit dem Stopptrick gearbeitet: Die Aufnahme wird bei gleichbleibendem Setting mehrmals gestoppt und die Szene verändert, indem sich wie durch Zauberhand Gegenstände bewegen oder Personen auftauchen bzw. verschwinden. Der Computer wurde überwiegend als Schnittstelle zu anderen Medienbereichen verwendet und diente mit spezieller Software der Überspielung, Speicherung und Bearbeitung von Fotos sowie von Audio- und Videodateien. Auch Lernsoftware wie das Sprachlernprogramm Schlaumäuse lernten die Erzieher/innen kennen. Bezogen auf Baackes (1997, 98f.) Dimensionen von Medienkompetenz lag der Schwerpunkt der Fortbildung vor allem bei der Mediengestaltung, da insbesondere die aktivierenden und kreativen Möglichkeiten der Mediennutzung nachhaltig im Alltag der Kitas verankert werden sollten.

Das Ziel der Evaluation war es, das Fortbildungsprojekt Medienkompetenz-Kitas NRW zu dokumentieren und zu bewerten, so wie dies immer wieder von Experten/

2 Unter Medienhelden/innen werden die von Kinder präferierten Medienfiguren verstanden. Die Präferenz für bestimmte Lieblingsfiguren wird medienpädagogisch mit den Kindern reflektiert, um die Kinder in der Verarbeitung ihrer medialen Erlebnisse zu unterstützen und Hinweise auf aktuelle Entwicklungsthemen der Kinder zu erhalten (WDR 2010, 38; Eder et al. 2014, 85ff.; Neuß 2008, 490). 
innen gefordert wird (KBoM! 2011, 16f.). Das besondere Augenmerk lag auf dem subjektiven Stellenwert von Medienerziehung in den Einrichtungen auf Seiten der Erzieher/innen, dem Selbstvertrauen der Erzieher/innen in der Handhabung von technischen Medien, ihrer Handlungsfähigkeit bei der Umsetzung von Medienprojekten mit Kindern sowie bei Aspekten der Nachhaltigkeit.

Das Evaluationsdesign bestand aus einer Kombination quantitativer und qualitativer Methoden (Bortz/Döring 2003). In die Evaluationsergebnisse sind unterschiedliche Instrumente eingeflossen: Vor Projektbeginn wurden die Einrichtungsleitungen ( $N=12)$ zur Situation und zur Relevanz von Medienerziehung in ihren jeweiligen Einrichtungen befragt. Die Erzieher/innen befragten sich unter Anleitung gegenseitig mittels eines Fragebogens (Partnerbefragung; $N=105$ ) zu ihren Haltungen und Erfahrungen hinsichtlich Medienerziehung und -nutzung und ihren Erwartungen an das Projekt. Die fünf Medienpädagogen/innen wurden telefonisch zu ihren Erwartungen an das Projekt befragt. Während des Projekts dokumentierten die Medienpädagogen/innen ihre Aktivitäten und Erfahrungen in Protokollbögen, die im Anschluss inhaltsanalytisch ausgewertet wurden. Zusätzlich wurden in zwei Kitas die medienpädagogischen Fortbildungen mittels passiver teilnehmender Beobachtung evaluiert. Nach Projektende wurden die Medienpädagogen/innen in einer Gruppendiskussion ( $\mathrm{N}=5$ ) zur Einschätzung des Projektes befragt. Die KitaLeitungen wurden telefonisch qualitativ zur Projekteinschätzung befragt $(N=12)$; zudem alle Kita-Mitarbeiter/innen mittels quantitativem Fragebogen ( $N=93$ ).

\section{Evaluationsergebnisse}

Die medienpädagogische Ausgangslage der Kitas entspricht vor Projektbeginn etwa den Befunden, die schon in anderen Erhebungen ermittelt wurden (etwa Six und Gimmler 2007a). Die Mediennutzung konzentriert sich auf traditionelle Medien wie Bücher. Die Unterversorgung mit technischen Geräten wie einem Computer wird von den Kita-Mitarbeiter/innen als zentrales Argument für die wenigen medienpädagogischen Aktivitäten in den Einrichtungen benannt. Die Erzieher/ innen schätzen im Rahmen der Partnerbefragung ihre subjektive Medienkompetenz im Umgang mit elektronischen Medien durchschnittlich mit der Schulnote befriedigend ein. Insgesamt 50 Prozent der Befragten bewerten ihre subjektive Medienkompetenz mit der Schulnote befriedigend, während 27 Prozent sich als ausreichend oder mangelhaft medienkompetent bezeichnen. Somit schätzt nur ein kleiner Teil der Befragten die subjektive Medienkompetenz als (sehr) gut ein. Bei der Frage nach dem Verständnis von Medienerziehung benennt über die Hälfte der Befragten (54\%) die Vermittlung eines kompetenten und verantwortungsvollen Umgangs mit Medien. Zudem sollen aus Sicht der Erzieher/innen den Kindern Grundkenntnisse im Umgang mit Medien vermittelt werden (21\%) (z. B. technische Handhabe des Computers als Vorbereitung auf schulische Anforderungen) und die Kinder an einen altersgerechten Umgang mit Medien (17\%) herangeführt werden. 
Gut die Hälfte der Einrichtungen hat bereits medienpädagogische Projekte durchgeführt. Gleichwohl verfügt kaum eine Einrichtung über eine Verankerung medienpädagogischer Anteile in ihrer pädagogischen Konzeption ${ }^{3}$.

Nach Beendigung der Fortbildung wurde eine abschliessende quantitative Befragung der Fortbildungsteilnehmer/innen durchgeführt. Fast 70 Prozent der Teilnehmer/innen sind mit der Konzeption und Durchführung der Fortbildung vollkommen zufrieden, ihre Erwartungen an das Projekt wurden insofern erfüllt. Besonders positiv wird bewertet, dass das gesamte Team einbezogen und dadurch gegenseitige Unterstützung ermöglicht wurde und dass das Fortbildungskonzept individuell an die Bedürfnisse und Erwartungen der Kitas angepasst wurde. Neben einem Zuwachs an eigenen technischen Fähigkeiten sehen die Fortbildungsteilnehmer/ innen nach Projektende auch eine Steigerung in Bezug auf ihre subjektive medienpädagogische Kompetenz (Abb. 1).

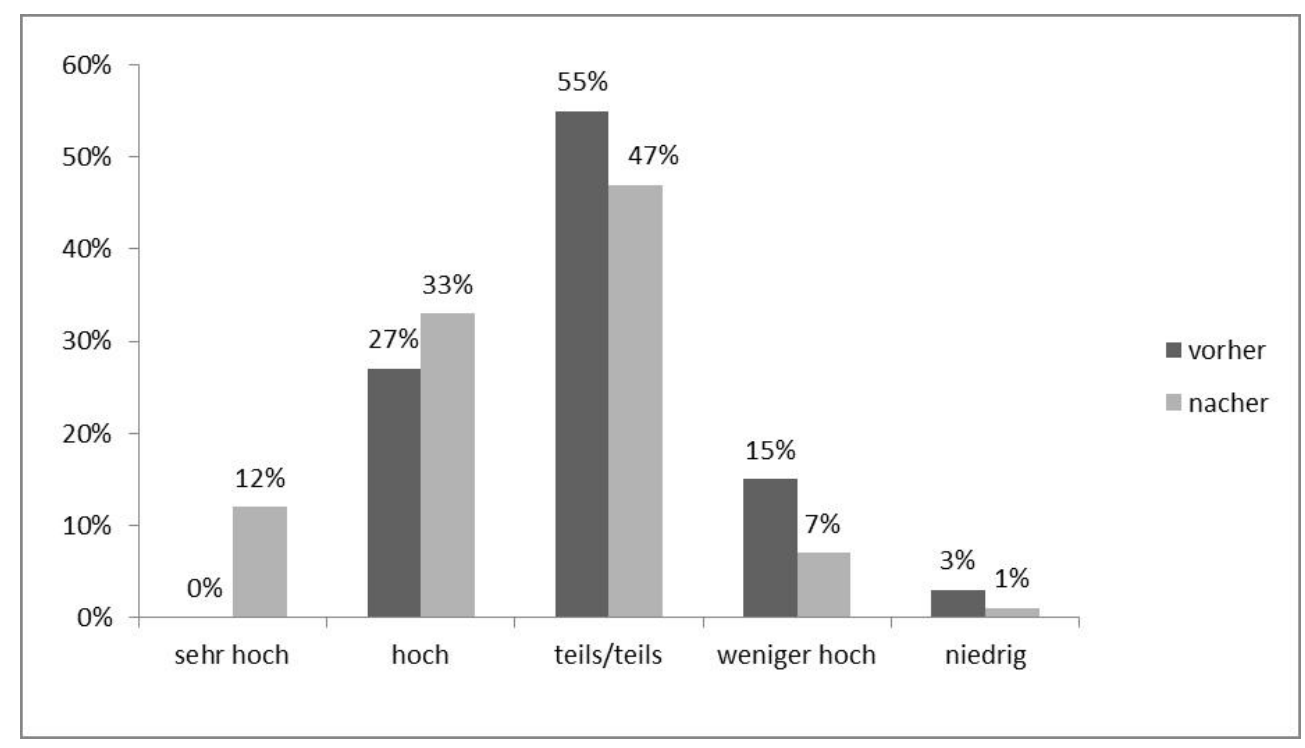

Abbildung 1: Subjektive medienpädagogische Kompetenz ${ }^{4}$

So schätzen nach Projektende 12 Prozent der Erzieher/innen ihre subjektive medienpädagogische Kompetenz als sehr hoch ein (gegenüber 0 Prozent bei der

3 Gemäss §22a, Abs. 1 SGB VIII sind die Träger einer Kindertagesstätte dazu verpflichtet, eine pädagogische Konzeption für die Einrichtung zu erstellen, an der die pädagogische Arbeit ausgerichtet wird. Ein solches Konzept spiegelt das pädagogische Leitbild der Einrichtung wider und soll die Qualität der Förderung sicherzustellen. Es beschreibt pädagogische Schwerpunkten und Ziele, die Zusammenarbeit mit den Eltern, Massnahmen zur Evaluation der pädagogischen Arbeit etc.

4 Partnerbefragung Kita-Mitarbeiterinnen zu Projektbeginn ( $n=98$ ); Quantitativer Fragebogen an Kitas zum Projektende ( $n=90)$; Die subjektive medienpädagogische Kompetenz wurde operationalisiert durch die subjektive eigene Qualifikation der Erzieher/innen zur Medienerziehung und ihr subjektiven Selbstvertrauens, in Zukunft selbstständig Medienprojekte in der Einrichtung durchzuführen. 
Anfangserhebung) und weitere 33 Prozent (im Gegensatz zu 27 Prozent bei der Anfangserhebung) bezeichnen diese als hoch.

Auch die subjektive Medienkompetenz gestaltet sich aus Sicht der Beteiligten nach Projektabschluss deutlich positiver als vor Beginn des Projekts: Durchschnittlich bewerten sich die Erzieherinnen nach Projektabschluss mit der Schulnote 2,44 (gut) im Vergleich zu einer 3,08 (befriedigend) zu Projektbeginn. Einige Erzieherinnen hatten zu Projektbeginn deutliche Scheu und nur wenig Erfahrung im Umgang mit Mediengeräten - vor allem mit dem Computer. Gegen Ende des Projekts liess sich eine Verbesserung bezüglich der eigenen Nutzungskompetenz erkennen. Die Scheu, die vor Beginn des Projekts im Umgang mit Mediengeräten bestand, konnte durch eigenständiges aktives Ausprobieren abgebaut und die technische Nutzungskompetenz gesteigert werden. Der Umgang mit den Medien wurde sentspannter> und technische Schwierigkeiten nahmen zum Ende des Projekts hin ab, wie die abschliessenden Interviews mit den Kita-Leitungen aufzeigen:

[...] da hat sich ganz deutlich ne Veränderung im Zugang zu Medien ergeben. Die Kolleginnen sind wesentlich offener und bereiter, dadurch, dass sie damit, also an den, an der Videokamera arbeiten konnten, mit Foto arbeiten konnten, am PC verschiedene Dinge machen, ausprobieren konnten; sind sicherer, sind dadurch auch wesentlich bereiter geworden, diese Dinge in den Alltag zu integrieren und auch mit Kindern im Alltag zu integrieren. (Kita-Leitung der Kita 9)

Dennoch zeigen sich auch nach Projektabschluss Unterschiede in der technischen Handhabung von Medien unter den Kita-Mitarbeiter/innen und damit einhergehend auch Unterschiede bezüglich der medienpädagogischen Aktivitäten. So schätzen 18 bzw. 16 Prozent der beteiligten Kita-Mitarbeiterinnen im Rahmen der quantitativen Abschlussbefragung ihre eigene Medienkompetenz nach wie vor in puncto Mediengestaltung und -nutzung mit der Schulnote ausreichend oder schlechter ein. Vor diesem Hintergrund erscheinen kontinuierliche Fortbildungsprozesse als äusserst sinnvoll.

Betrachtet man die medienpädagogische Praxis in den Einrichtungen nach Projektende werden der Computer und vor allem die Digitalkamera nun deutlicher in die pädagogische Arbeit integriert. Die Digitalkamera wird nicht mehr wie bisher nur im Rahmen der Bildungsdokumentation gemäss des Kinderbildungsgesetzes (KiBiz) eingesetzt, sondern darf nun überwiegend selbstständig von den Kindern genutzt werden. Zur langfristigen Verankerung der Medienerziehung in den Kitas haben alle beteiligten Kitas Anschaffungen getätigt bzw. planen weitere Anschaffungen (häufig Erwerb von Digitalkameras und digitalen Bilderrahmen; in mehreren Kitas Anschaffung des Kinderaufnahmegeräts «Easispeak»; Gestaltung von PC-Räumen etc.). Viele Einrichtungen verbinden ihre Medienarbeit (wie auch teilweise bereits vor Projektbeginn) mit anderen Themenschwerpunkten wie Sprachförderung oder 
auch Naturwissenschaft und Technik, um weitere Bildungsbereiche abzudecken und Kompetenzen unterschiedlicher Art zu schulen. Einige Einrichtungen planen zudem feste Medienaktivitäten: Beispielsweise wird eine Kita einmal in der Woche das Thema Fernsehhelden/innen mit den Kindern besprechen, eine Einrichtung wird die medienpädagogischen Aktivitäten der Kinder im Rahmen eines medienpädagogischen Aktivitätenspiegels wöchentlich dokumentieren und eine weitere, medienpädagogisch bereits erfahrene Einrichtung wird das Thema Medienarbeit weiterhin in die pädagogische Projektarbeit einbinden: Während die älteren Kinder selbst kleine Filme drehen, mit den Erzieher/innen Vor- und Abspann gestalten und Musik hinterlegten, stünde bei jüngeren Kindern eher der Handlungsaspekt im Vordergrund (z. B. sich von den Erziehern/-innen mit einer Marionette filmen zu lassen).

Die Teilnahme an der Fortbildung brachte keine prinzipielle Veränderung in der Einschätzung des Stellenwerts von Medienerziehung in der Kita mit sich. Medienerziehung stellt nach Abschluss des Projekts für ca. 20 Prozent der Kita-Mitarbeiter/innen ein sehr wichtiges (vor Projektbeginn: 18\%) und für 61 Prozent der Erzieherinnen ein wichtiges Thema (vor Projektbeginn: 65\%) dar. Gleichwohl möchten alle Kitas - so die Ergebnisse der leitfadengestützten Interviews mit den Kita-Leitungen nach Projektabschluss - nach der Beendigung des Projektes weiterhin medienpädagogische Aktivitäten durchführen und fühlen sich dazu auch grundsätzlich in der Lage. Teilweise wurde von den Kita-Leitungen dezidiert der Wunsch geäussert, weiterhin von dem/r bekannten Medienpädagogen/in betreut zu werden, um eine nachhaltige Verankerung der Ansätze zu gewährleisten.

Immerhin 60 Prozent der Kita-Mitarbeiter/innen nehmen sich nach Projektende als (sehr) motiviert wahr, selbstständig nach Abschluss des Projekts ein Medienprojekt durchzuführen (Abb. 2).

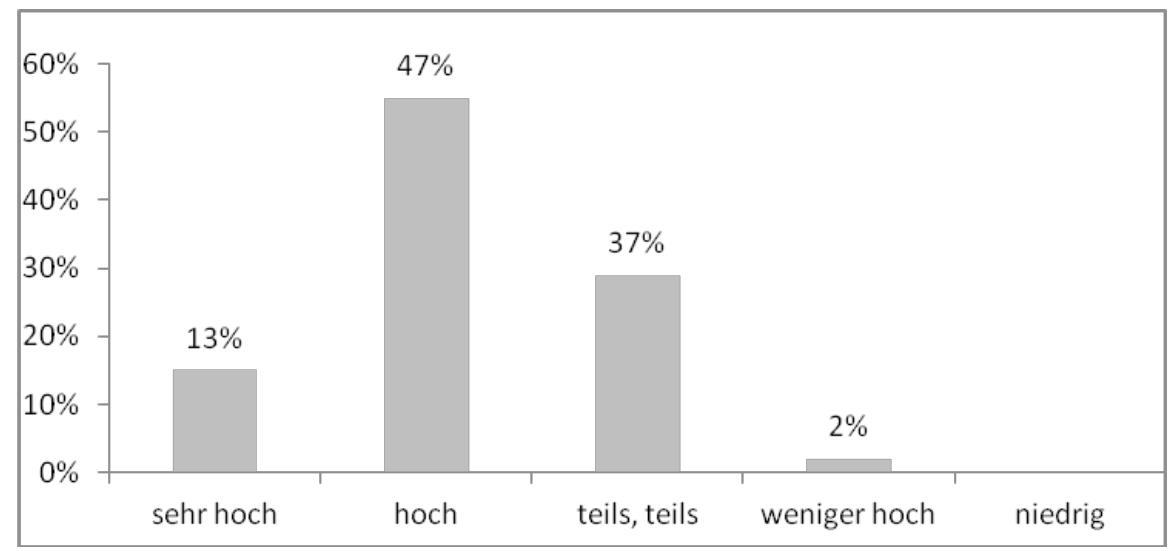

Abbildung 2: Subjektive Motivation bzgl. der zukünftigen selbstständigen Durchführung eines Medienprojekts ${ }^{5}$

5 Quantitativer Fragebogen an Kitas zum Projektende $(n=91)$ 
Die Motivation zur Durchführung eigener Medienprojekte resultiert vermutlich auch aus den Rückmeldungen, die die Erzieher/innen während der Durchführung der begleiteten Medienprojekte von den Kindern und Eltern erhielten. Die KitaKinder zeigten sich laut den passiven teilnehmenden Beobachtungen im Projekt offen und interessiert, neugierig und sehr aktiv. Sie waren mit Spass und grösstenteils auch hoher Konzentration bei der Sache, waren experimentierfreudig und stellten viele Fragen. Auch die Eltern schätzten die Aktivitäten nach Einschätzung der Kita-Leitungen als sehr positiv ein.

Dennoch bestehen auch nach der Fortbildung aus Sicht der Kita-Mitarbeiter/innen weiterhin Hemmnisse zur Umsetzung von Medienerziehung. Diese unterscheiden sich kaum von den Antworten bei Projektbeginn. Dabei werden nach wie vor insbesondere drei zentrale Faktoren, die Medienerziehung behindern, genannt: Zwei Hemmnisse beziehen sich auf strukturelle Rahmenbedingungen: Zum einen werden knappe Zeitressourcen genannt, die laut den Abschlussinterviews mit den Kita-Leitungen unter anderem mit den Anforderungen des Kinderbildungsgesetzes und dem Bildungsauftrag der Kitas begründet werden. Zum anderen sehen die Kita-Mitarbeiter/innen immer noch einen Mangel an geeigneten Mediengeräten. Zudem benennen die Teilnehmer/innen nach wie vor eine fehlende subjektive Qualifikation zur Medienerziehung, was verdeutlicht, dass trotz der relativ langen Laufzeit dieses spezifischen Projekts weitere medienspezifische Fördermassnamen erforderlich sind. Dementsprechend bestätigen die Ergebnisse der Evaluation empirische Daten weiterer Studien (mpfs 2015, 29; Meister et al. 2012, 19ff., 22; Six/Gimmler 2007a, 132ff., 148ff., 192ff.; Institut für Demoskopie Allensbach 2015, 36ff.).

Aus den Evaluationsergebnissen geht zudem hervor, dass in den meisten Kitas vor der Fortbildung keine medienpädagogischen Inhalte im pädagogischen Konzept der Einrichtung verankert waren bzw. nur einige Zeilen zum Thema Medienerziehung existierten. Durch das Projekt hat ein Grossteil der Kitas während bzw. im Anschluss an das Projekt begonnen, ihr schriftlich fixiertes Einrichtungskonzept um medienpädagogische Inhalte zu erweitern. Das Projektziel, gemeinsam mit den Medienpädagogen/innen ein medienpädagogisches Leitbild zu erstellen, wurde aufgrund mangelnder Projektzeit jedoch nicht vollständig erreicht, zumindest wurde ein solches in vielen Fällen (noch) nicht schriftlich fixiert.

Im Hinblick auf die zeitliche Dauer des Projekts ist festzuhalten, dass insgesamt 27 Prozent der Kita-Mitarbeiter/innen sowie die im Projekt zuständigen Medienpädagogen/innen knappe Zeitressourcen bemängelten.

\section{Implikationen}

Die Ergebnisse empirischer Studien verdeutlichen, dass der Bildungsbereich Medien bisher nur vereinzelt in Kindertagesstätten umgesetzt wird. Dementsprechend werden die Chancen, die eine vorschulische Medienerziehung für eine kind- 
liche Heranführung an eine reflektierte Mediennutzung bietet, nicht ausreichend genutzt. Auf der Grundlage vorliegender Studien sowie der Einbeziehung der Evaluationsergebnisse der Projektinitiative Medienkompetenz-Kitas NRW werden deshalb im Folgenden Schlussfolgerungen für eine nachhaltige Verankerung von Medienerziehung in Kindertageseinrichtungen gezogen. Hierbei sollen sowohl Implikationen im Hinblick auf Fortbildungen von Erzieher/innen als auch Implikationen im Hinblick auf die Erzieher/in-Ausbildung und frühkindliche Studiengänge aufgezeigt werden.

\section{Implikationen im Hinblick auf Fortbildungen von Erzieher/innen}

- Medienpädagogische Inhouse-Schulungen mit Theorie- und Praxisphasen:

Die Initiative Medienkompetenz-Kitas NRW verdeutlicht den Vorteil von InhouseSchulungen, in denen das gesamte Kita-Team einbezogen wird, gegenüber zentralisierten Fortbildungen, die nur die Leitungen oder einzelne Erzieher/innen adressieren und somit das Risiko eines mangelnden Wissenstransfers an das restliche Kita-Team innehaben. Durch die Verankerung von Schnuppertagen, bei denen die Kita-Mitarbeiter/innen inhaltliche, technische und konzeptionelle Fragen mit den Medienpädagogen/innen für ihren Arbeitsalltag erarbeiten (ohne Anwesenheit der Kinder) und Thementagen mit Anwesenheit der Kinder konnten sich die Kollegen/innen gemeinsam in ihrer Einrichtung an das Thema Medienerziehung herantasten. Dementsprechend bietet sich eine solche Aufteilung in Theorie- und Praxisphasen in medienpädagogischen Fortbildungen an.

- Individualisierte Fortbildungen:

Es hat sich gezeigt, dass eine Flexibilität des Fortbildungspersonals, auf die Gegebenheiten vor Ort einzugehen, sich als sehr hilfreich erweist. Es sollte in Fortbildungen darauf geachtet werden, dass diese sowohl eng an die Bedürfnislagen der Erzieher/innen und Kinder sowie die Bedingungen vor Ort anknüpfen. Hierbei sollte den Kitas zeitliche Freiräume gewährt werden, damit sie die Fortbildung ihren Präferenzen entsprechend in den Kita-Alltag integrieren können. So kann den Einrichtungen bspw. freigestellt werden, ob sie eine Projektwoche durchführen oder einzelne Termine für die Fortbildung wählen.

- Ausreichender zeitlicher Rahmen:

Medienpädagogische Praxisprojekte benötigen einen ausreichenden zeitlichen Rahmen. So war im vorliegenden Projekt nicht in allen Einrichtungen ausreichend Zeit, ein medienpädagogisches Konzept zu verschriftlichen und eine Reflexion des Projekts mit dem/r Medienpädagogen/in durchzuführen. Dies erscheint indes im Hinblick auf eine nachhaltige Verankerung von Medienerziehung als zwingend erforderlich.

- Qualifizierung der Medienpädagogen/innen im Vorfeld:

Eine Qualifizierung der Medienpädagogen/innen wie sie im Vorfeld von Medienkompetenz Kitas NRW stattgefunden hat, ist empfehlenswert. Hierbei sollten so- 
wohl Inhalte auf technischer und medienpraktischer Ebene als auch auf personaler Ebene (Moderation etc.) eingeübt und vertieft werden. Von einer solchen Qualifizierung dürften auch erfahrenere Medienpädagogen/innen profitieren, wenn diese gezielt auf die Einrichtungsform (in diesem Fall Kindertagesstätten) abgestimmt ist.

- Verknüpfung von Medienerziehung mit anderen Bildungsbereichen:

Die vorliegenden empirischen Studien sowie die Evaluation von Medienkompetenz-Kitas NRW zeigen auf, dass sowohl die ausgebildeten Erzieher/innen in den Einrichtungen als auch die angehenden Erzieher/innen, die sich noch in der Ausbildung befinden, eine Knappheit an zeitlichen Ressourcen wahrnehmen. Gebündelt mit einer meist höheren Relevanzwahrnehmung anderer Bildungsbereiche spielt Medienerziehung in der Kita - wenn überhaupt - meist nur eine marginale Rolle (siehe u.a. Institut für Demoskopie Allensbach 2015, 19; Friedrichs 2013, 9). Daran ansetzend sollten sowohl in Aus- und Fortbildung Möglichkeiten aufgezeigt werden, Medienerziehung mit anderen Bildungsbereichen gewinnbringend zu verknüpfen (z.B. durch ein gemeinsames Fotografieren von Kindern in der Natur o.ä.). - Medienpädagogische Elternarbeit:

Damit die medienpädagogische Arbeit in den Kitas nachhaltig gelingt, erscheint es unabdingbar, auch die Eltern in die medienpädagogische Arbeit einzubinden, da die Familie den zentralen Ort der frühkindlichen Medienerfahrung darstellt. Dementsprechend sollten Fortbildungen diesen Bereich der Medienerziehung einbeziehen (bspw. in Form von medienpädagogischen Elternabenden).

- Weitere medienpädagogische Unterstützung:

Zur langfristigen Verankerung von Medienerziehung im Kita-Alltag benötigen die Erzieher/innen auch nach Projektende weitere Unterstützung, da im Alltag immer wieder sowohl technische als auch pädagogische Fragen aufkommen. Aufgrund dessen ist es ratsam, dass die aus einer Fortbildung bekannten Medienpädagogen/innen auch nach Abschluss einer Fortbildungseinheit weiterhin zur Verfügung stehen, um je nach Bedarf den Kitas bei medienbezogenen Fragen weiter zu helfen und regelmässig medienpädagogische Handlungsanregungen anzubieten.

- Modell-Kitas als Hospitationsort:

Kitas, die bereits medienpädagogisch «fit) sind, sollten als Modell-Kitas agieren, die einen Hospitationsort für weitere interessierte Kitas darstellen. Gerade in diesem Kontext erscheint eine weitere medienpädagogische Unterstützung solcher Modell-Kitas unabdingbar.

- Flächendeckende Fortbildungen unter Bereitstellung ausreichender finanzieller Ressourcen:

Medienpädagogische Fortbildungen sind kostenspielig, vor allem, wenn es sich um Inhouse-Schulungen handelt, da diese mit höheren Fortbildungs- und Reisekosten als bei einer eintägigen Veranstaltung an einem zentralisierten Fortbil- 
dungsort verbunden sind. Um Medienerziehung langfristig zu verankern, dürfen diese Kosten nicht auf Seiten der Einrichtungen bzw. der Träger verortet sein. Stattdessen bedarf es breit angelegter geförderter Projekte, die flächendeckend umgesetzt werden (Friedrichs und Gross 2013, 292f.). Hiermit kann auch der Problematik entgegengewirkt werden, dass sich vor allem diejenigen, die eh für das Thema Medienerziehung in der Kita offen sind, mit der Thematik auseinandersetzen (Marci-Boehncke et al. 2012, 8f.), während Erzieher/innen mit eher abwehrenden Haltungen, sei es, da andere Bereiche als relevanter für die Kita-Arbeit empfunden werden und/oder negative medienbezogene Wirkannahmen bestehen, den Bildungsbereich Medienerziehung nicht kennenlernen.

- Bereitstellung ausreichender Mediengeräte in den Einrichtungen:

Im Rahmen produktionsorientierter Medienarbeit sind Mediengeräte unabdingbar. In diesem Zusammenhang ist eine Ausstattung der Einrichtungen mit Tablets als Geräten, die verschiedenste Medienfunktionen bereitstellen (fotografieren, filmen, Nutzung von Apps etc.) anzustreben. Sinnvoll ist hierbei eine flächendeckende Medienausstattung wie in den 1990er Jahren im Projekt Schulen ans Netz e.V. im Kontext der schulischen Internetausstattung.

- Bündelung von Akteuren/innen der (Medien)erziehung:

Um eine breite Verankerung von Medienerziehung in Kindertagesstätten zu erzielen, ist es notwendig, frühzeitig alle bedeutsamen Bereiche - die Fachkräfte, die Träger von Einrichtungen und Fortbildungen, die Partner/innen in den Sozialräumen (Fachberatungen, Familienbildungsstätten etc.) und die politische Ebene - einzubeziehen, um eine gemeinsame Zielstellung einer frühkindlichen Medienerziehung zu entwickeln (siehe auch Six/Gimmler 2007a, 296).

Implikationen im Hinblick auf die Erzieher/in-Ausbildung und frühkindliche Studiengänge Als mindestens ebenso bedeutsam im Vergleich zu Fortbildungsmassnahmen erscheinen Implikationen auf der Ebene der Erzieher/innen-Ausbildung, da diese den «Grundstein [...] für die spätere medienpädagogische Kompetenz und für die Motivation, sich in diesem Bereich zu engagieren» (Six und Gimmler 2007b, 4) legt. Folgende Implikationen empfehlen die Autorinnen:

- Verankerung medienpädagogischer Inhalte in der Lehrer/innenbildung:

Six und Gimmler (2007a, 66f.) zeigen Defizite hinsichtlich der Qualifikation der Lehrkräfte, welche medienpädagogische Inhalte unterrichten, auf. Dementsprechend sind eine stärkere Verankerung medienpädagogischer Inhalte sowohl in den Studiengängen der Lehrer/innenbildung als auch in Fortbildungen für Lehrkräfte notwendig (Tulodziecki 2012).

- Mehr Zeitressourcen für die Auseinandersetzung mit elektronischen Medien durch die Koppelung von Inhalten bereitstellen:

Weiter sollte statt einer Trennung in die Bereiche Kinderliteratur (1. Ausbildungsjahr) und elektronische Medien/produktionsorientierte Medienarbeit (2. Ausbil- 
dungsjahr), wie es teilweise in Nordrhein-Westfalen geregelt ist, bereits im ersten Ausbildungsjahr der Medienbereich berücksichtigt werden. Hierbei bietet sich eine Verknüpfung beider Bereiche an (bspw. mittels einer Verfilmung oder Vertonung eines (Bilder-)Buches) (Friedrichs 2013, 12).

- Erhöhung medienpädagogischer Praxiserfahrungen:

Zentral ist zudem, dass die Auszubildenden medienpädagogische Praxiserfahrungen mit Kindern in Kitas sammeln und dass es nicht nur bei ‘Trockenübungens in den Fachschulen bleibt (Friedrichs 2013, 13). Dies ist auch insofern relevant, dass, worauf die Analyseergebnisse der Gruppendiskussionen von Auszubildenden durch Friedrichs hinweisen, positiv wahrgenommene Praxiserfahrungen der Auszubildenden ihre Bereitschaft zur Medienerziehung in der Kita und auch das Zutrauen in die eigene medienpädagogische Kompetenz fördern können.

- Kooperationen zwischen Fachschulen und Kindertagesstätten:

Gerade im Hinblick auf Modifikationen der Erzieher/innen-Ausbildung erscheinen zudem konkrete Kooperationen und Absprachen der Fachschulen mit Kindertagesstätten sowie eine Ausweitung an Projektmöglichkeiten sinnvoll, sodass medienpädagogische Projektideen in der Praxis umgesetzt werden können (ebd., 13).

- Verankerung medienpädagogischer Inhalte in Studiengängen der frühkindlichen Bildung:

Neben der klassischen Erzieher/innen-Ausbildung wurden in den letzten Jahren verschiedene Studiengänge der frühkindlichen Bildung an Universitäten und Fachhochschulen eingeführt. Im Hinblick auf medienpädagogische Praxiserfahrungen ist es zum einen problematisch, dass nicht alle Studiengänge medienpädagogische Inhalte umfassen (WiFF o.J.) und zum anderen, dass die Praktikumszeiten im Vergleich zur klassischen Erzieher/innen-Ausbildung teilweise kürzer sind (Blossfeld et al. 2012, 59). Im Rahmen einer Neu- und Re-Konzeptionalisierung von Studiengängen der frühkindlichen Bildung ist der Bereich der Medienerziehung unbedingt zu berücksichtigen (KBoM! 2013).

Wie der vorliegende Artikel aufzeigt, bedarf es noch weitreichender Anstrengungen, um Medienerziehung nachhaltig und flächendeckend zu verankern. Dazu bedarf es neben mehr Kooperationen der verschiedenen Akteure/innen in einem breiten Netzwerk vor allem weiterer bildungspolitischer Initiativen.

\section{Literatur}

Aufenanger, Stefan. 2013. «Digitale Medien im Leben von Kindern zwischen null und fünf Jahren.» merz 57 (2): 8-14.

Aufenanger, Stefan und Franz Gerlach. 2005. Vorschulkinder und Computer: Sozialisationseffekte und pädagogische Handlungsmöglichkeiten in Tageseinrichtungen für Kinder. 18.5.2015. http://www.lpr-hessen.de/files/Forschungsbericht _Versionlnternet.pdf 
Baacke, Dieter. 1997. Grundlagen der Medienkommunikation. Bd. 1: Medienpädagogik. Tübingen: Niemeyer.

Bachmair, Ben. 2002. «Der Pädagoge als Gärtner? Die Gestaltung der Lebenswelt mit Hilfe von Medienerziehung.» nexus 7: 12-13.

Becker, Ulrike. 2015. «Medien und Geschlecht.»In Medienpädagogik - ein Überblick, hrsg. v. Friederike von Gross, Dorothee M. Meister und Uwe Sander, 306322. Weinheim und Basel: Beltz Juventa.

Blossfeld, Hans-Peter, Wilfried Bos, Hans-Dieter Daniel, Bettina Hannover, Dieter Lenzen, Manfred Prenzel et al. 2012. Professionalisierung in der Frühpädagogik: Qualifikationsniveau und -bedingungen des Personals in Kindertagesstätten. Gutachten. 1. Aufl. Münster: Waxmann.

Bortz, Jürgen und Nicola Döring. 2003. Forschungsmethoden und Evaluation für Human- und Sozialwissenschaftler. 3. Aufl. Berlin u.a.: Springer.

Brüggemann, Marion, Ines Averbeck und Andreas Breiter. 2013. Förderung von Medienkompetenz in Bremer Kindertageseinrichtungen: Bestandsaufnahme und Befragung von Fachkräften in Bremen und Bremerhaven zur frühen Medienbildung. 18.5.2005. http://www.bremische-landesmedienanstalt.de/fileadmin/user_upload/content/dateien/broschueren/Meko-Kita-Sept2013_ifib.pdf.

Dreyer, Stefan, Claudia Lampert und Anne Schulze. 2014. Kinder und Onlinewerbung: Erscheinungsformen von Werbung im Internet, ihre Wahrnehmung durch Kinder und ihr regulatorischer Kontext. Berlin: Vistas.

Eder, Sabine, Anke Lehmann, André Lenich, Susanne Roboom, Gerhard Seilerund Johannes Wentzel. 2014. Medienkompetenz-Kitas NRW:Ein ModellprojektderLandesanstalt für Medien Nordrhein-Westfalen. 22.02.2015. http://www.meko-kitas -nrw.de/fileadmin/kita-nrw/user_upload/download/L155_MedienkompetenzKitas-NRW3124844174.pdf.

Eder, Sabine und Susanne Roboom. 2014. «Klicken, Knipsen, Tricksen ... Medienerziehung im Kindergarten.» In Handbuch Kinder und Medien, hrsg. v. Angela Tillmann, Sandra Fleischer u. Kai-Uwe Hugger, 503-516. Wiesbaden: Springer VS.

Egmont Ehapa. 2013. KidsVerbraucherAnalyse 2013. Berlin.

Feierabend, Sabine und Walter Klingler. 2014. «Was Kinder sehen: Eine Analyse der Fernsehnutzung Drei- bis 13-Jähriger 2013.» media perspektiven 4: 182-194.

Friedrichs, Henrike. 2013. "Der medienerzieherische Habitus angehender Erzieherlnnen und Bedingungen für die Ausübung von Medienerziehung in Kindertagesstätten.» medienimpulse 4. 18.5.2015. http://www.medienimpulse.at /articles/view/611.

Friedrichs, Henrike und Friederike von Gross. 2013. «Erzieherischer Jugendmedienschutz: Medienpädagogische Initiativen und Projekte.» In Jugendmedien- 
schutz in Deutschland, hrsg. v. Henrike Friedrichs, Thorsten Junge u. Uwe Sander, 257-294. Wiesbaden: Springer VS.

Friedrichs, Henrike und Meister, Dorothee M. 2015. «Medienerziehung in der Kindertagesstätte.» In Medienpädagogik - ein Überblick, hrsg. v. Friederike von Gross, Dorothee M. Meister u. Uwe Sander, 273-305. Weinheim und Basel: Beltz Juventa.

Gesetz zur frühen Bildung und Förderung von Kindern. (Kinderbildungsgesetz KiBiz). Viertes Gesetz zur Ausführung des Kinder- und Jugendhilfegesetzes SGB VIII -. Vom 30. Oktober 2007. Fassung vom 01.08.2011.

Götz, Maya. 2010. «Was nebenbei noch so hängen bleibt. Oder die Frage, wie Menschen vom Fernsehen lernen, ohne es zu merken.» Televizion 23 (1): 6-11.

Götz, Maya. 2014. «Sexy Girls in Hotpants. Die Hypersexualisierung im Kinderfernsehen.» In MedienConcret 14 (1): 30-33.

Götz, Maya und Lemish, Dafna, Hrsg. 2012. Sexy Girls, Heroes and Funny Losers. Gender Representation in Children's TV around the World. München: Peter Lang.

Hentig, Hartmut von. 1987. "Werden wir die Sprache der Computer sprechen? Der pädagogische Aspekt.» Neue Sammlung: Vierteljahres-Zeitschrift für Erziehung und Gesellschaft 27 (1): 69-85.

Hoffmann, Dagmar. 2004. «Zum produktiven Umgang von Kindern und Jugendlichen mit medialen Identifikationsangeboten.» merz 6: 7-91.

Institut für Demoskopie Allensbach. 2015. Digitale Medienbildung in Grundschule und Kindergarten. Ergebnisse einer Befragung von Elter, Lehrkräften an Grundschulen und Erzieher(innen) in Kindergärten im Auftrag der Deutsche Telekom Stiftung. 18.5.2015. http://www.telekom-stiftung.de/dts-cms/sites/default/files //dts-library/materialien/pdf/ergebnisse_allensbach-umfrage_gesamt.pdf.

Keine Bildung ohne Medien! (KBoM!), Hrsg. 2011. Bildungspolitische Forderungen: Medienpädagogischer Kongress 2011. Ludwigsburg.

Keine Bildung ohne Medien! (KBoM!) 2013. Keine Bildung ohne Medien! Offener Brief. Forderungen zur Medienkompetenzförderung anlässlich der aktuellen Koalitionsverhandlungen. 31. Oktober2013.18.05.2015. http://www.keine-bildungohne-medien.de/wp-content/uploads/2013/10/OffenerBrief_Koalitionsver handlung_Medienkompetenzf\%C3\%B6rderung.pdf.

Keine Bildung ohne Medien! (KBoM!) 2014. Initiative «Keine Bildung ohne Medien!» fordert: Grundbildung Medien für alle pädagogischen Fachkräfte. 18.5.2015. http://www.keine-bildung-ohne-medien.de/wp-content/uploads/2014/06 /Position_Grundbildung_KBoM.pdf

Krotz, Friedrich. 2003. «Medien als Ressource der Konstitution von Identität: Eine konzeptionelle Klärung auf der Basis der Symbolischen Interaktionismus.» In Medienidentitäten, Identität im Kontext von Globalisierung und Medienkultur, 
hrsg. v. Carsten Winter, Tanja Thomas u. Andreas Hepp. 27-48. Köln: von Halem.

Kunczik, Michael und Zipfel, Astrid. 2013. «Mediengewalt in Film und Fernsehen.» In Jugendmedienschutz in Deutschland, hrsg. v. Henrike Friedrichs, Thorsten Junge u. Uwe Sander, 299-302. Wiesbaden: Springer VS.

Livingstone, Sonia und Ellen J. Helsper. 2006. «Does advertising literacy mediate the effects of advertising on children? A critical examination of two linked research literatures in relation to obesity and food choice.» Journal of Communication 56: 560-584.

Luca, Renate. 2010. «Mediale Körperrepräsentationen und Rezeptionspraktiken des doing gender am Beispiel von Germany's Next Topmodel.» In Fokus Medienpädagogik: Aktuelle Forschungs- und Handlungsfelder, hrsg. v. Petra Bauer et al., 208-220. München: kopaed.

Marci-Boehncke, Gudrun. 2008. «Medienerziehung in der KiTa - Kompetenzen und Meinungen der Erzieherinnen.» Ludwigsburger Beiträge zur Medienpädagogik 11.

Marci-Boehncke, Gudrun, Rath, Matthias und Anita Müller. 2012. «Medienkompetent zum Schulübergang: Erste Ergebnisse einer Forschungs- und Interventionsstudie zum Medienumgang in der Frühen Bildung.» MedienPädagogik 22 (27. Dez.): 1-22. www.medienpaed.com/22/\#marci-boehncke1212.

Medienpädagogischer Forschungsverbund Südwest (mpfs). 2012. FIM-Studie 2011: Familie, Interaktion \& Medien. Untersuchung zur Kommunikation und Mediennutzung in Familien. Stuttgart. 18.5.2014. http://www.mpfs.de/fileadmin /FIM/FIM2011.pdf

Medienpädagogischer Forschungsverbund Südwest (mpfs) 2015. miniKim 2014: Kleinkinder und Medien; Basisuntersuchung zum Medienumgang 2- bis 5-Jähriger. 18.5.2015. http://www.mpfs.de/fileadmin/miniKIM/2014/miniKIM_2014.pdf.

Meister, Dorothee M. 2013. «Vermittlung von Medienkompetenz in der Praxis für Kinder und Jugendliche: Schule.» In Medienkompetenzförderung für Kinder und Jugendliche: Eine Bestandsaufnahme, hrsg. v. Bundesministerium für Familie, Senioren, Frauen und Jugend, 46-52. Niestetal: Silber Druck OHG.

Meister, Dorothee M., Henrike Friedrichs, Karolina Keller, Anja Pielsticker und Timon Tobias Temps. 2012. Chancen und Potenziale digitaler Medien zur Umsetzung des Bildungsauftrags in Kindertageseinrichtungen in NRW: Forschungsbericht der Gesellschaft für Medienpädagogik und Kommunikationskultur (GMK) und der Universität Paderborn. 18.5.2015. http://kw1.uni-paderborn.de /fileadmin/mw/Meister/Projeke/Kitas_NRW_bericht_2012.pdf

Meister, Dorothee M., Henrike Friedrichs, Sonja Kröger und Uwe Sander. 2011. «Förderung von Werbekompetenz bei Kindern: Eine Bestandsaufnahme medienpädagogischer Materialien. Werbung ohne Grenzen: Wie können beste- 
hende Konzepte zur Förderung der Werbekompetenz von Kindern optimiert werden?» medienimpulse 4. 19.5.2015. http://www.medienimpulse.at/articles /view/355

Ministerium für Schule und Weiterbildung des Landes Nordrhein-Westfalen (MSW NRW). 2014. Richtlinien und Lehrpläne. Fachschulen des Sozialwesens. Fachrichtung Sozialpädagogik.

Ministerium für Schule und Weiterbildung des Landes Nordrhein-Westfalen/Ministerium für Generationen, Familie, Frauen und Integration des Landes Nordrhein-Westfalen (MSW NRW/MGFFI NRW). 2010. Mehr Chancen durch Bildung von Anfang an - Entwurf - Grundsätze zur Bildungsförderung für Kinder von 0 bis 10 Jahren in Kindertageseinrichtungen und Schulen im Primarbereich in Nordrhein-Westfalen.

Möller, Ingrid und Krahé, Barbara. 2013. "Gewalt in Bildschirmspielen und aggressives Verhalten.»In Jugendmedienschutz in Deutschland, hrsg. v. Henrike Friedrichs, Thorsten Junge u. Uwe Sander, 309-313. Wiesbaden: Springer VS.

Neuß, Norbert. 2008. "Medienbildung und Bildung im Kindergarten.» In Handbuch Medienpädagogik, hrsg. v. Uwe Sander, Friederike von Gross und KaiUwe Hugger, 489-497. Wiesbaden: VS.

Neuß, Norbert. 2013. «Medienkompetenz in der frühen Kindheit.»In Medienkompetenzförderung für Kinder und Jugendliche: Eine Bestandsaufnahme, hrsg. v. Bundesministerium für Familie, Senioren, Frauen und Jugend, 34-45. Berlin.

Pfeiffer, Christian. 2011. "Computerspielen als Ursache von Schulversagen? Was ist zu tun?» In Hätte Kant gesurft? Wissen und Bildung im Internet-Zeitalter, hrsg. v. Rolf Wernstedt u. Marei John-Ohnesorg, 22-34. Berlin: Friedrich-EbertStiftung.

Rogge, Jan-Uwe. 2007. «Kinder brauchen Helden: Einige Gedanken.»Televizion 20 (2): 50-53.

Schorb, Bernd. 2005. "Medienerziehung.» In Grundbegriffe Medienpädagogik, hrsg. v. Jürgen Hüther u. Bernd Schorb, 240-243. 4. vollst. neu konzipierte Aufl. München: kopaed.

Six, Ulrike, Christoph Frey und Roland Gimmler. 1998. Medienerziehung im Kindergarten: Theoretische Grundlagen und empirische Befunde. Bd. 28. Opladen: LfR-Schriftenreihe Medienforschung.

Six, Ulrike und Roland Gimmler. 2007a. Förderung von Medienkompetenz im Kindergarten: Eine empirische Studie zu Bedingung und Handlungsformen der Medienerziehung. Bd. 57. Opladen: LfM-Schriftenreihe Medienforschung.

Six, Ulrike und Roland Gimmler. 2007b. Förderung von Medienkompetenz im Kindergarten: Eine empirische Studie zu Bedingungen und Handlungsformen der Medienerziehung. 19.5.2015. https://www.lfm-nrw.de/fileadmin/lfm-nrw/Presse meldungen/medienkom-kiga-zusamm.pdf. 
Sozialgesetzbuch (SGB). Achtes Buch (VIII). Kinder- und Jugendhilfe.

Spitzer, Manfred. 2012. Digitale Demenz: Wie wir uns und unsere Kinder um den Verstand bringen. Droemer: München.

Tudodziecki, Gerhard. 2012. «Medienpädagogische Kompetenz und Standards in der Lehrerbildung.» In Jahrbuch Medienpädagogik 9, hrsg. v. Renate SchulzZander, Birgit Eickelmann, Heinz Moser, Horst Niesyto u. Petra Grell, 271-297. Wiesbaden: VS.

Vollbrecht, Ralf. 2010. «Werbung und Konsum.»In Handbuch Mediensozialisation, hrsg. v. Ralf Vollbrecht u. Claudia Wegener, 316-323. Wiesbaden: VS.

WDR. Hrsg. 2010. Radio- und Fernsehprojekte für den Kindergarten: Die Medienwelt erkunden mit der Bärenbude (WDR5) und der Sendung mit dem Elefanten (WDR Fernsehen und KI.KA). Arbeitspaket mit Materialien für Erzieherinnen und Erzieher.

Weiterbildungsinitiative Frühpädagogische Fachkräfte (WiFF) (o.J.). Studium. Studiengangsdatenbank. 19.5.2015. www.fruehpaedagogik-studieren.de. 